\title{
Research on Risk Assessment of the Transformer Based on Life Cycle Cost
}

\author{
Hui Zhou ${ }^{\mathrm{a}}$, Guowei Wu' ${ }^{\mathrm{a}}$, Weiwei Pan ${ }^{\mathrm{a}}$, Yunhe Hou ${ }^{\mathrm{b}}$, Chong Wang ${ }^{\mathrm{b} *}$ \\ ${ }^{a}$ Zhejiang Electric Power Corporation, Hangzhou, China \\ ${ }^{b}$ Department of Electrical and Electronic Engineering, The University of Hong Kong, Hong Kong, China
}

\begin{abstract}
This paper proposes an optimization model of life cycle cost $(L C C)$ of the transformer. First, to build the model of $L C C$ of the transformer, the whole $L C C$ of the transformer is divided into five costs, i.e., initial cost, operating cost, maintenance cost, fault cost and disposal cost. The whole $L C C$ is calculated. Second, according to the data of several kinds of transformers, the relationship between the initial cost and the whole $L C C$ is established by using the curve fitting method. The optimal initial cost that minimizes the whole $L C C$ is calculated. Third, risk sources of $L C C$ are identified and associated risk index is established based on the proposed model. To quantify risks, the fuzzy analytic hierarchy process (FAHP) method is used to establish the index of the cost of each stage. According to the index, the risks can be sorted based on the importance. The degree of risks of $L C C$ can also be obtained according to the index.
\end{abstract}

Keywords: Life cycle cost, optimal cost, risk assessment, risk index

\section{Introduction}

Appropriate life cycle management [1-2] is important to ensure the safety and efficiency of the whole transmission systems [3-5]. The transformer is one of the important components in transmission systems. Therefore, the optimal life cycle cost (LCC) of the transformer in its life time will play an important role in the whole life cycle cost management of transmission systems.

This paper proposes an optimization model of life cycle cost of the transformer. Based on the proposed model, risk sources are identified and associated risk index is established. Some risk management methods for $L C C$ are established.

The work of the paper is organized as follows. To build the model of LCC of the transformer, the whole LCC process of the transformer is divided into five costs, i.e., initial cost, operating cost, maintenance cost, fault treatment cost and disposal cost. Each cost is analyzed, thus the whole LCC is calculated. According to the data of transformers provided by Zhejiang Electric Power Corporation of State Grid Corporation of China, the relationship between the initial cost and the whole LCC can be simulated by using the curve fitting method. And the optimal initial cost that makes the whole LCC minimum can be calculated. Critical factors, which affect the cost of each stage, are identified. The fuzzy analytic hierarchy process (FAHP) [6-8] is employed to establish the index system of each cost to analyze the risks quantitatively. According to the index, the risks can be sorted based on the importance and some risk management control methods can be proposed to reduce the cost risk. The $L C C$ model and the index system have been successfully applied in the transmission system of Zhejiang Electric Power Corporation of State Grid Corporation of China.

\footnotetext{
* Manuscript received July 15, 2012; revised August 15, 2012.

Corresponding author. Tel.: +852-28578422; E-mail address: tonywangchong@163.com.
} 


\section{Optimization Model of Life Cycle Cost of Transformers}

\subsection{Model of life cycle cost}

The whole $L C C$ of transformers is divided into five costs, i.e., initial cost $C_{I}$, operating cost $C_{O}$, maintenance cost $C_{M}$, fault cost $C_{F}$ and disposal cost $C_{D}$.

Initial cost refers to the cost paid in the duration of planning and construction of the transformer. It includes the design cost, the transformer purchase cost, construction and installation cost. The design cost mainly includes the research cost, the cost of feasibility study, preliminary design cost, and so on.

Operating cost refers to the sum of all costs that are needed during the operation period of the transformer. It mainly includes the loss cost, operating labor cost and so on. The operating cost of each year can be described as follows:

$$
C_{O}=C_{\text {loss }}+C_{P}
$$

where $C_{P}$ is the operating labor cost, $C_{\text {loss }}$ is the cost caused by the power loss of the transformer, it can be calculated as:

$$
C_{\text {loss }}=8760 \times P_{\text {loss }} \times \eta \times a
$$

where $P_{\text {loss }}$ is the load loss of the transformer, $\eta$ is the load rate of the transformer, $a$ is the electricity price.

Maintenance cost refers to the maintenance staff cost, the repair cost and so on. The data of the cycle of repair and overhaul and the cost of each repair and overhaul can be provided by the manufacturers, so maintenance cost can be calculated according to the cycle of repair and overhaul and the cost of each repair and overhaul.

Fault cost is the economic loss cost caused by failure outage. Unplanned Outage Coefficient (UOC) can be used to calculate the fault cost of each year approximately. It can be described as follows:

$$
C_{F}=U O C \times P_{r} \times \eta \times a
$$

where $P_{r}$ is the rated active power of the transformer.

Disposal cost refers to the cost needed in the decommissioning process. In addition to the payment of the necessary remedial treatment cost, some component salvage can be recycled. The total disposal cost can be considered as a proportion of the purchase cost approximately.

The whole $L C C$ can be calculated as follows:

$$
L C C=C_{I}+\sum_{i=1}^{N}\left(\frac{1+r}{1+R}\right)^{i-1} C_{O i}+\sum_{i=1}^{N}\left(\frac{1+r}{1+R}\right)^{i-1} C_{M i}+\sum_{i=1}^{N}\left(\frac{1+r}{1+R}\right)^{i-1} C_{F i}+\left(\frac{1+r}{1+R}\right)^{N-1} C_{D}
$$

where $r$ is the inflation rate, $R$ is the annual interest rate, $N$ is the life cycle of the transformer, $C_{O i}$ is the operating cost of the $i$ th year, $C_{M i}$ is the maintenance cost of the $i$ th year, $C_{F i}$ is the fault cost of the $i$ th year.

\subsection{Optimization model of LCC}

The whole $L C C$ of the transformer can be calculated according to the above analysis. Because lower initial cost and higher cost may both result in high $L C C$, an appropriate initial cost is important to make the whole $L C C$ minimum.

The function between the initial cost and the whole LCC, which can be calculated according to the equations (1) (4), is built by using the curve fitting method.

Table 1 shows the parameters of six cases provided by Zhejiang Electric Power Corporation of State Grid Corporation of China. The life cycle of the transformer is 30 years. The electricity cost is 0.5 $\mathrm{RMB} / \mathrm{kWh}$, the disposal coefficient is approximately $10 \%$, the annual interest rate is $4 \%$ and the inflation rate is $6 \%$. 
Table 1. Parameters of Each Case

\begin{tabular}{|l|c|c|c|c|c|c|}
\hline \multicolumn{1}{|c|}{ Parameters } & Case 1 & Case 2 & Case 3 & Case 4 & Case 5 & Case 6 \\
\hline$C_{I}\left(\times 10^{6} \mathrm{RMB}\right)$ & 10 & 10.75 & 11.90 & 13.40 & 15.10 & 17.00 \\
\hline$P_{\text {loss }}(\mathrm{kW})$ & 550 & 510 & 450 & 420 & 410 & 400 \\
\hline$\eta$ & 0.6 & 0.6 & 0.6 & 0.6 & 0.6 & 0.6 \\
\hline$C_{P}\left(\times 10^{6} \mathrm{RMB}\right)$ & 0.10 & 0.086 & 0.071 & 0.057 & 0.050 & 0.042 \\
\hline $\mathrm{UOC}(\%)$ & 0.018 & 0.162 & 0.015 & 0.0135 & 0.012 & 0.011 \\
\hline$P_{r}(\mathrm{MVA})$ & 180 & 180 & 180 & 180 & 180 & 180 \\
\hline Cycle of Repair $($ Year $)$ & 3 & 3 & 3 & 5 & 5 & 5 \\
\hline Cost of Each Repair $\left(\times 10^{6} \mathrm{RMB} /\right.$ Year $)$ & 0.08 & 0.10 & 0.12 & 0.25 & 0.30 & 0.35 \\
\hline Cycle of Overhaul $($ Year $)$ & 6 & 6 & 6 & 10 & 10 & 10 \\
\hline Cost of Each Overhaul $\left(\times 10^{6} \mathrm{RMB} /\right.$ Year $)$ & 0.80 & 1.00 & 1.20 & 2.20 & 2.50 & 3.00 \\
\hline
\end{tabular}

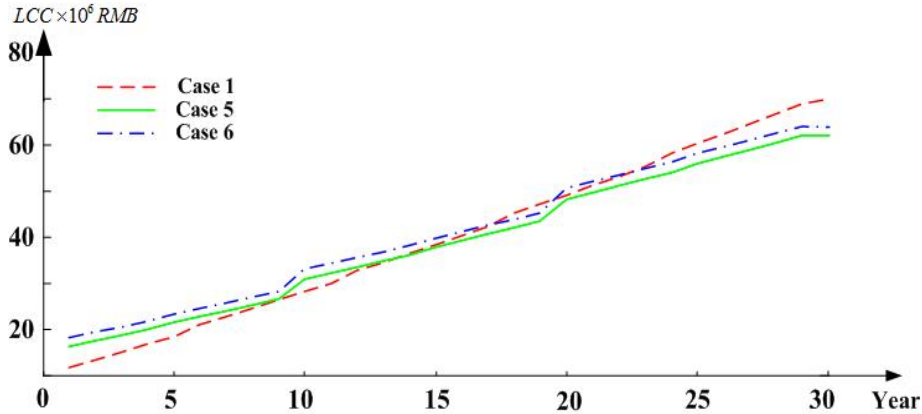

(a)

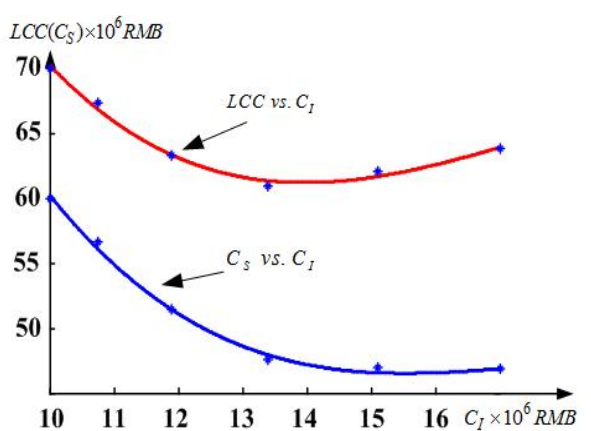

(b)

Fig. 1. (a) $L C C$ curves of case 1 , case 5 and case 6; (b) Curves of $L C C$ vs. $C_{I}$ and $C_{S}$ vs. $C_{I}$

Fig. 1 (a) shows $L C C$ curves of case 1, case 5 and case 6 . According to the Fig. 1 (a), case 6 has the maximum initial cost, but its whole $L C C$ is not the maximal. Case 1 has the minimum initial cost, but its whole $L C C$ is the maximal. So the initial cost is an important factor of the whole $L C C$. According to the provided initial costs and the whole $L C C$ s of six cases, the relationship between the initial cost $\left(C_{I}\right)$ and the whole $L C C$ can be simulated by:

$$
L C C=-4 \times 10^{-6} C_{I}^{3}+2.1 \times 10^{-2} C_{I}^{2}-35.01 C_{I}+2.5 \times 10^{4}
$$

According to the equation (5), the optimal initial cost can be calculated and its value is $13.96 \times 10^{6}$ RMB. In the practice, the initial cost which is nearest to the $13.96 \times 10^{6}$ RMB can be considered as the optimal strategy. Fig. 1 (b) shows the curves of $C_{I}$ vs. $L C C$ and $C_{I}$ vs. $C_{S} . C_{S}$ can be described as follows:

$$
C_{S}=C_{O}+C_{M}+C_{F}+C_{D}
$$

\section{Analysis of the Risks of $L C C$}

There are many uncertainties in the whole life cycle. The $L C C$ of components will be affected by these uncertainties. To analyze these uncertainties quantitatively, risk resources of life cycle cost are identified and then the fuzzy analytic hierarchy process is employed to establish a risk assessment model.

\subsection{Risks of LCC}

According to the five costs mentioned in section 2, the LCC risks (F) based on these five costs include initial cost risks (S1), operating cost risks (S2), maintenance cost risks (S3), fault cost risks (S4) and disposal cost risks (S5). 
Initial cost risks mainly include the price risk (T11), the technological progress risk (T12), the investigation risk (T13), the load forecasting risk (T14), the component location risk (T15) and the personal quality risk (T16).

Operating cost risks mainly include the operation risk (T21), the load control risk (T22), the legal risk (T23) and the management risk (T24).

Maintenance cost risks mainly include the uncertainty of maintenance risk (T31), the operation risk (T32) and the management risk (T33).

Fault cost risks mainly include the failure number risk (T41), the load loss risk (T42) and the personal quality risk (T43).

Disposal cost risks mainly include the disposal application risk (T51), the disposal approval risk (T52), the inactive facilities statistic risk (T53) and the inactive facilities disposal risk (T54).

\subsection{Principle of FAHP}

AHP is a multi-attribute decision-making model proposed by Saaty [6]. This method is widely used to solve decision-making problem with uncertainties due to its advantages of integral structure, simple theory and easy operation. However, AHP has a disadvantage of the ambiguity in decision making. Fuzzy analytic hierarchy process (FAHP) is proposed by Laarhoved and Pedrycz [8] to overcome this disadvantage.

In the process of $F A H P$, a paired comparison matrix is built by using the evaluation criteria, as shown in the judgment matrix $A$ :

$$
A=\left[a_{i j}\right]=\left[\begin{array}{cccc}
1 & a_{12} & \cdots & a_{1 n} \\
a_{21} & 1 & \cdots & \vdots \\
\vdots & \vdots & \ddots & \vdots \\
a_{n 1} & a_{n 2} & \cdots & 1
\end{array}\right]
$$

where $a_{i j}$ can be determined by the 1-9 scale [9] . A is a reciprocal matrix with $a_{i j}>0, a_{i j}=1 / a_{j i}$, $a_{i i}=1$.

$$
I_{C}=\frac{\lambda_{\max }-n}{n-1}, \quad R_{C}=\frac{I_{C}}{R_{R}}
$$

where $I_{C}$ and $R_{C}$ are the consistency index and the consistency ratio respectively, $n$ is the dimension of matrix and $\lambda_{\max }$ is the largest eigenvalue of $A, R_{R}$ is the random consistency ration shown in Table 2 . When $R_{C} \geq 0.1$, the matrix $A$ should be rectified appropriately.

Table 2. The Value of $R_{R}$

\begin{tabular}{cccccccccc}
\hline$n$ & 1 & 2 & 3 & 4 & 5 & 6 & 7 & 8 & 9 \\
\hline$R_{R}$ & 0 & 0 & 0.52 & 0.89 & 1.12 & 1.26 & 1.36 & 1.41 & 1.46 \\
\hline
\end{tabular}

The establishment of alternative set shows as follows:

$$
V=\left\{v_{1}, v_{2}, v_{3}, v_{4}, v_{5}\right\}=\text { (very low, low, medium, high, very high) }
$$

where $v_{i}$ represents the risk degree of the critical factors.

During the establishment of the fuzzy relationship matrix, the fuzzy relationship $E$ can be obtained through expert assessments. When evaluate the fuzzy comprehensive, the second-level fuzzy comprehensive evaluation vector $D_{i}$ can be calculated as follows:

$$
D_{i}=w_{i} \cdot E_{i}
$$


Then the comprehensive evaluation matrix $D$ can be formed by $D_{i}$. The evaluation result can be calculated by multiplying the $D$ by the weight vector $w$ :

$$
H=w \cdot D
$$

\subsection{Evaluation of risks of $L C C$}

Based on the risks of $L C C$, a serial of judgment matrix of relative importance between criteria can be defined as according to the equation (7):

$$
\begin{aligned}
& F-S=\left(\begin{array}{ccccc}
1 & 3 & 1 & 1 / 5 & 5 \\
1 / 3 & 1 & 1 / 3 & 1 / 5 & 3 \\
1 & 3 & 1 & 1 / 3 & 3 \\
5 & 5 & 3 & 1 & 7 \\
1 / 5 & 1 / 3 & 1 / 5 & 1 / 7 & 1
\end{array}\right), \quad S 2-T=\left(\begin{array}{cccc}
1 & 5 & 7 & 3 \\
1 / 5 & 1 & 3 & 1 / 3 \\
1 / 7 & 1 / 3 & 1 & 1 / 3 \\
1 / 3 & 3 & 3 & 1
\end{array}\right), \quad S 4-T=\left(\begin{array}{ccc}
1 & 5 & 3 \\
1 / 5 & 1 & 1 / 3 \\
1 / 3 & 3 & 1
\end{array}\right) \\
& S 1-T=\left(\begin{array}{cccccc}
1 & 1 / 3 & 1 / 7 & 1 / 3 & 1 / 5 & 3 \\
3 & 1 & 1 / 5 & 1 & 1 & 3 \\
7 & 5 & 1 & 3 & 3 & 5 \\
3 & 1 & 1 / 3 & 1 & 1 & 3 \\
5 & 1 & 1 / 3 & 1 & 1 & 3 \\
1 / 3 & 1 / 3 & 1 / 5 & 1 / 3 & 1 / 5 & 1
\end{array}\right), S 3-T=\left(\begin{array}{ccc}
1 & 5 & 3 \\
1 / 5 & 1 & 1 / 3 \\
1 / 3 & 3 & 1
\end{array}\right), \quad S 5-T=\left(\begin{array}{cccc}
1 & 1 & 1 / 3 & 1 / 5 \\
1 & 1 & 1 / 3 & 1 / 5 \\
3 & 3 & 1 & 1 / 3 \\
5 & 5 & 3 & 1
\end{array}\right)
\end{aligned}
$$

\begin{tabular}{|c|c|c|}
\hline Level F-Level S & Local weight of sub-criteria & Global weight \\
\hline \multirow{6}{*}{$\begin{array}{c}\text { F-S1 } \\
\text { Weight }=0.1803\end{array}$} & $\mathrm{~T} 11=0.0671$ & 0.0121 \\
\hline & $\mathrm{T} 12=0.1390$ & 0.0251 \\
\hline & $\mathrm{T} 13=0.4314$ & 0.0778 \\
\hline & $\mathrm{T} 14=0.1491$ & 0.0269 \\
\hline & $\mathrm{T} 15=0.1663$ & 0.0300 \\
\hline & $\mathrm{T} 16=0.0472$ & 0.0085 \\
\hline \multirow{4}{*}{$\begin{array}{c}\text { F-S2 } \\
\text { Weight }=0.0870\end{array}$} & $\mathrm{~T} 21=0.5688$ & 0.0495 \\
\hline & $\mathrm{T} 22=0.1280$ & 0.0111 \\
\hline & $\mathrm{T} 23=0.0659$ & 0.0057 \\
\hline & $\mathrm{T} 24=0.2372$ & 0.0206 \\
\hline \multirow{3}{*}{$\begin{array}{c}\text { F-S3 } \\
\text { Weight }=0.1945\end{array}$} & $\mathrm{~T} 31=0.6333$ & 0.1232 \\
\hline & $\mathrm{T} 32=0.2605$ & 0.0206 \\
\hline & $\mathrm{T} 34=0.1062$ & 0.0507 \\
\hline \multirow{3}{*}{$\begin{array}{c}\text { F-S4 } \\
\text { Weight }=0.4955\end{array}$} & $\mathrm{~T} 41=0.6687$ & 0.3314 \\
\hline & $\mathrm{T} 42=0.0882$ & 0.0437 \\
\hline & $\mathrm{T} 43=0.2431$ & 0.1205 \\
\hline \multirow{4}{*}{$\begin{array}{c}\text { F-S5 } \\
\text { Weight }=0.0427\end{array}$} & $\mathrm{~T} 51=0.0825$ & 0.0035 \\
\hline & $\mathrm{T} 52=0.0814$ & 0.0035 \\
\hline & $\mathrm{T} 53=0.2715$ & 0.0116 \\
\hline & $\mathrm{T} 54=0.5646$ & 0.0241 \\
\hline
\end{tabular}

According to FAHP, overall criteria weights can be calculated and shown in Table 3:

Table 3. Weights of the Evaluation Index

According to expert evaluations, the fuzzy relation between evaluation factors and remarks set $V$ can be expressed as evaluation matrix $E$. Take the evaluation of the risk of $C_{I}$ as an example. The single factor evaluation matrix $E_{1}$ is: 
The weight of the risk of $C_{I}$ is:

$$
E_{1}=\left(\begin{array}{ccccc}
0.1 & 0.4 & 0.2 & 0.2 & 0.1 \\
0.1 & 0.2 & 0.4 & 0.2 & 0.1 \\
0.1 & 0.5 & 0.2 & 0.1 & 0.1 \\
0.2 & 0.5 & 0.2 & 0.1 & 0 \\
0.4 & 0.4 & 0.1 & 0.1 & 0.1 \\
0.1 & 0.4 & 0.3 & 0.2 & 0
\end{array}\right)
$$

$$
w_{1}=(0.0671,0.1390,0.4314,0.1490,0.1663,0.0472)
$$

Then, the single-factor evaluation result set $D_{1}$ can be denoted as:

$$
D_{1}=w_{1} E_{1}=(0.1648,0.4302,0.2159,0.1253,0.0804)
$$

Other evaluation results also can be calculated by the same method.

$$
\begin{array}{ll}
D_{2}=(0.1237,0.4047,0.2256,0.1697,0.0763), & D_{3}=(0.1000,0.4048,0.2212,0.1740,0.1000) \\
D_{4}=(0.1000,0.4067,0.2176,0.1757,0.1000), & D_{5}=(0.1565,0.4673,0.2163,0.1164,0.0435)
\end{array}
$$

The comprehensive evaluation can be calculated:

$$
\begin{aligned}
H & =w \times D=\left(\begin{array}{l}
0.1803 \\
0.0870 \\
0.1945 \\
0.4955 \\
0.0427
\end{array}\right)^{T} \times\left(\begin{array}{l}
0.1648,0.4302,0.2159,0.1253,0.0804 \\
0.1237,0.4047,0.2256,0.1697,0.0763 \\
0.1000,0.4048,0.2212,0.1740,0.1000 \\
0.1000,0.4067,0.2176,0.1757,0.1000 \\
0.1565,0.4673,0.2163,0.1164,0.0435
\end{array}\right) \\
& =(0.1162,0.4130,0.2187,0.1632,0.0920)
\end{aligned}
$$

According to the comprehensive evaluation $H$, the maximum index is 0.4130 and the risk of $L C C$ of the transformer is low based on the principle of the maximum membership degree. According to the global weight in Table 3, the risks can be sorted based on the importance. For critical risks, the corresponding measures can be adopted to reduce the risks of the $L C C$.

\section{Conclusion}

The paper divides the whole $L C C$ into five costs, i.e., initial cost, operating cost, maintenance cost, fault cost and disposal cost according to the characteristic of the transformer. An optimization model of $L C C$ is built. The cost of each stage is analyzed, thus the whole $L C C$ can be calculated. The relationship between the initial cost and the whole $L C C$ can be simulated by using the curve fitting method. Based on the proposed model, risk sources are identified and associated risk index is established. To quantify the risk, a fuzzy analytic hierarchy process method is used to establish the index of the cost of each stage, and the risk degree of $L C C$ of the transformer can be obtained according to the index. A realistic case study from Zhejiang Electric Power Corporation of State Grid Corporation of China is reported to validate the proposed method.

\section{Acknowledgements}

This research is supported by Zhejiang Electric Power Corporation of State Grid Corporation of China and Research Institute of HKU in Shenzhen.

\section{References}

[1] Asset Management Part 1: Specification for the Optimized Management of Physical Infrastructure Assets, PAS 55-1 (UK) 2008. 
[2] Asset Management Part 2: Specification for the Optimized Management of Physical Infrastructure Assets, PAS 55-2 (UK) 2008.

[3] Takehara A, Tanaka H, Shibata T, Nagata, M Kurihara. A new life-cycle risk map (LCRM) for developing maintenance strategies for transmission equipment. In: Proc. of IEEE Power and Energy Society General Meeting, 2008:1-7.

[4] Allahmanli L, Chattopadhyay G, Edwards G. Challenges of asset management in power transmission network. In: Proc. of IEEE International Conference on Industrial Engineering and Engineering Management, 2008:561-565.

[5] Mohapatra R., Mukhopadhyay S. Risk and asset management of transmission system in a reformed power sector. Presented at: 2006 IEEE Power India Conference.

[6] T. L. Saaty. The Analytic Hierarchy Process. New York:McGraw-Hill; 1980.

[7] Huang Y, Huang WJ. Analysis of the life cycle cost of the power plants based on analytic hierarchy process method. In: Proc. of International Conference on Information Management, Innovation Management and Industrial Engineering, 2009:639-642.

[8] Laarhoven P, Pedrycz W. A fuzzy extension of Satty’s priority theory. Fuzzy Sets and System, 1983; 11(1):229-241.

[9] Wu FD, Hu NL. Study on the model of safety evaluation in coal mine based on Fuzzy-AHP comprehensive evaluation method. Presented at: 2011 International Conference on Mechatronic Science, Electric Engineering and Computer. 